\title{
Kinematic gait analysis of workers exposed to knee straining postures by Bayes decision rule
}

\author{
Neila Mezghani*1,2,3, Nathaly Gaudreault ${ }^{4}$, Amar Mitiche ${ }^{5}$, Leila Ayoubian ${ }^{6}$, Youssef Ouakrim ${ }^{1}$, Nicola $^{2}$ \\ Hagemeister 2,3 , Jacques A Deguise ${ }^{2,3}$ \\ ${ }^{1}$ LICEF, Télé-université (TELUQ), Montreal, Canada \\ ${ }^{2}$ Laboratoire de recherche en imagerie et orthopédie, Centre de recherche du CHUM, Montreal, Quebec, Canada \\ ${ }^{3}$ École de technologie supérieure, ÉTS, Montreal, Quebec, Canada \\ ${ }^{4}$ École de réadaptation, Sherbrooke University, Quebec, Canada \\ ${ }^{5}$ Institut National de la Recherche Scientifique, INRS-EMT, Montreal, Quebec, Canada \\ ${ }^{6}$ Aberdeen Biomedical Imaging Center, Aberdeen University, Aberdeen, United Kingdom
}

Received: May 30, 2015

DOI: $10.5430 /$ air.v4n2p106
Accepted: June 17, 2015

Online Published: July 13, 2015

\begin{abstract}
Deep knee flexion postures such as kneeling and squatting have been demonstrated, in recent review of occupational knee disorders, as a risk factor of developing knee osteoarthritis (OA). This study investigates a probabilistic method to analyze knee gait kinematics measurements of workers exposed to knee straining postures to determine if they are in any way similar to those of knee OA patients. The measurements we use are clinically relevant kinematic signals, namely the variation during a locomotion gait cycle of the angles the knee makes with respect to the three-dimensional (3D) planes of flexion/extension, internal/external rotation, and abduction/adduction. Three groups of participants were used: a set of 24 workers exposed to knee straining postures (KS workers) acting as a test group, a control group of 25 non-KS posture workers, and a reference knee OA group of 29 subjects. We compared the kinematic data of KS workers to those of knee OA patients and non-KS subjects using the Bayes decision theory. The results shows that, using the 3D data taken together or the abduction/adduction data, the KS workers resembles often to the OA patients. The analysis on the transverse plane and on sagittal plane, i.e., the flexion/extension and the internal/external rotation, are not conclusive as the similarities are not significant. The kinematic gait analysis by Bayes decision rule shows the similarity of workers exposed to knee straining postures to OA gait pattern and justifies further prospective studies of KS workers in order to assess if gait pattern could be modified even before the onset of the disease.
\end{abstract}

Key Words: Bayesian decision rule, Biomechanics, Kinematic gait analysis, Knee osteoarthritis, Knee straining postures

\section{Introduction}

Osteoarthritis is the most common type of musculoskeletal disorder and the knee is one of the most affected joints. ${ }^{[1]}$ A review of the literature highlighted that pathogenesis of osteoarthritis is multi factorial. Joint injury ${ }^{[1]}$ and physical activity $^{[2]}$ are among the leading risk factors in the develop- ment and progression of the disease. ${ }^{[3]}$

Epidemiological studies have shown an association between occupational activities and knee osteoarthritis (OA) development. ${ }^{[4-6]}$ Working in kneeling/squatting posture and lifting/carrying heavy weights have been identified as occupational risk factors. Moreover, these physically demanding

*Correspondence: Neila Mezghani; Email: neila.mezghani@teluq.ca; Address: Laboratoire de recherche en imagerie et orthopédie, Centre de recherche du CHUM, Montreal, Quebec, Canada. 
activities were shown to cause excessive mechanical loading on the knee joints, thereby increasing the risk of severe OA. ${ }^{[7]}$ These indicators have contributed some valuable information toward the diagnosis of OA, its risk factors, and its development. ${ }^{[8,9]}$ However, these studies have assessed the physical workload of workers based on self-evaluation or membership to typical occupations rather than by direct monitoring of knee function. Moreover, their focus was not specifically on the impact of posture on knee function.

Biomechanical gait measurements, which currently can be easily acquired in clinical settings, ${ }^{[10]}$ inform directly on knee function. They can be formally analyzed to determine factors influencing OA progression and thus can be used for the early detection early detection of people vulnerable to the disease, which makes it possible to change joint biomechanics before OA causes irreversible damage to the joint. For instance, some studies have been able to distinguish between asymptomatic subjects and knee OA using kinematic measurements. Other investigations could relate advanced stages of knee OA to ranges of knee joint rotation angles. ${ }^{[11-14]}$ In a practical setting, knee gait kinematics of workers exposed to knee straining postures were compared to those of workers who were not. The analysis highlighted some statistically significant differences on characteristic gait data, such as a greater knee flexion angle at initial foot contact and a lower peak knee flexion angle during the swing phase and a lower angle range for non-KS workers. ${ }^{[15]}$ Finally, other studies have reported that exposure to deep knee flexion resulted in changes in peak knee adduction and flexion moments as well as in peak flexion angles. ${ }^{[16]}$ However, no study has addressed explicitly the problem of determining the similarity between kinematic data of workers exposed to knee straining postures and kinematics of OA patients. A similarity with OA gait pattern would justify further prospective studies of KS (knee straining) workers, eventually showing that gait pattern of these workers could be modified even before the actual onset of the disease, as measured by X-Ray, for example.

The present study investigates a novel method which uses kinematic gait signal analysis to determine if workers exposed to knee straining postures have knee kinematics that resemble those of knee OA patients. Our basic hypothesis is that these workers have a signal gait patterns similar to those of knee OA patients rather than of non-knee straining workers. We used three groups of participants: a group of workers exposed to knee straining postures (KS workers) serving as a test group, a control group of workers not exposed to knee straining postures(non-KS workers), and a reference knee OA group. We examined three clinically relevant kinematic signals: flexion/extension, internal/external rotation, and abduction/adduction angle variations during a locomotion gait cycle. By Bayes decision rule, we confirmed our hypothesis that the gait patterns of the KS workers are similar to the patterns of OA patients rather than of non-KS.

\section{Method}

\subsection{Participants}

For a participation at large, all the subjects who took part in this study were recruited using advertisements in local newspapers in Montreal (Quebec, Canada). Fliers presenting information about the project were also distributed. Institutional ethics approval was obtained and all participants signed the informed consent.

The participants formed three distinct groups. One group is composed of 24 healthy workers (19 men and 5 women) with no diagnosis of clinical or radiological knee OA but who were exposed to occupational knee straining postures (KS). KS participants had to be 18 years of age or older and had to be exposed on a daily basis to sustained deep knee flexion postures while kneeling or squatting during working hours for at least $30 \mathrm{~min} /$ day and for at least the past five years. The second group is the control group, composed of 25 non-KS workers (12 men and 13 women) unexposed to occupational knee straining postures. Finally, the knee OA group contains 29 knee OA patients (17 men and 12 women). More details on the inclusion/exclusion criteria of the KS/non-KS group and of the OA patients can be found in Ref. ${ }^{[15]}$ and in Ref., ${ }^{[17]}$ respecively.

Three-dimensional (3D) knee kinematics signals with respect to the frontal, sagittal, and transverse planes were recorded from each participant while walking on a conventional treadmill at a self-selected comfortable speed. A knee marker attachment system, the KneeKG system, ${ }^{[10]}$ was installed on the participant's knee to record the 3D kinematics during two trials of $25 \mathrm{sec}$. This motion capture tool is composed of a harness and plate fixed quasi-rigidly onto the femoral condyles and tibial crest (see Figure 1), and provides accurate (0.4-2.3 and 1.1-2.4 mm), repeatable (0.4-0.8 and 0.8-2.2 mm), and reliable (intra class coefficient of 0.88 0.94) measurements. ${ }^{[10]}$ Fifteen representative gait cycles were averaged to obtain a mean pattern per subject based on an adjusted coefficient of multiple determinations. ${ }^{[18]}$ This was followed by interpolation and resampling from $1 \%$ to $100 \%$ of the gait cycle, therefore giving 100 measurement points for each participant. These data processing steps were performed using Matlab 2008b (The Mathworks, USA). To reduce the dimensionality of the data, we considered the first 20 gait cycle percentage points since these are generally considered the most relevant points for knee OA analysis. ${ }^{[19]}$

\subsection{Bayes decision rule}

The purpose of this analysis is to determine whether the KS workers have gait patterns similar to those of knee OA patients rather than of non-KS workers using Bayes classification. We examined three clinically relevant kinematic curve measurements: flexion/extension (sagittal plane), internal/external (transverse plane), and abduction/adduction 
(frontal plane) angle variations during a locomotion gait cycle. The analysis was conducted for the three planes together by concatenation of the gait cycle data into a single vector. We also performed the same analysis for each separate plane data to look at the behavior of the data for each plane independently of the other two. The analysis can be summarized as follows:

Let $\omega_{1}$ designate the non-KS workers group, and $\omega_{2}$ the knee OA patients group. Let $\mathrm{x}$ be the feature vector. For the frontal plane data, for instance, $x$ is a 20 element vector composed of the first 20 abduction/adduction angle measurements of the gait cycle. The Bayes formula provides the a posteriori probability of measurement $\mathrm{x}$ to be from group $\omega_{i}, i \in\{1,2\}$ :

$$
p\left(\omega_{i} \mid \mathrm{x}\right)=\frac{p\left(\mathrm{x} \mid \omega_{i}\right) p\left(\omega_{i}\right)}{p(\mathrm{x})}
$$
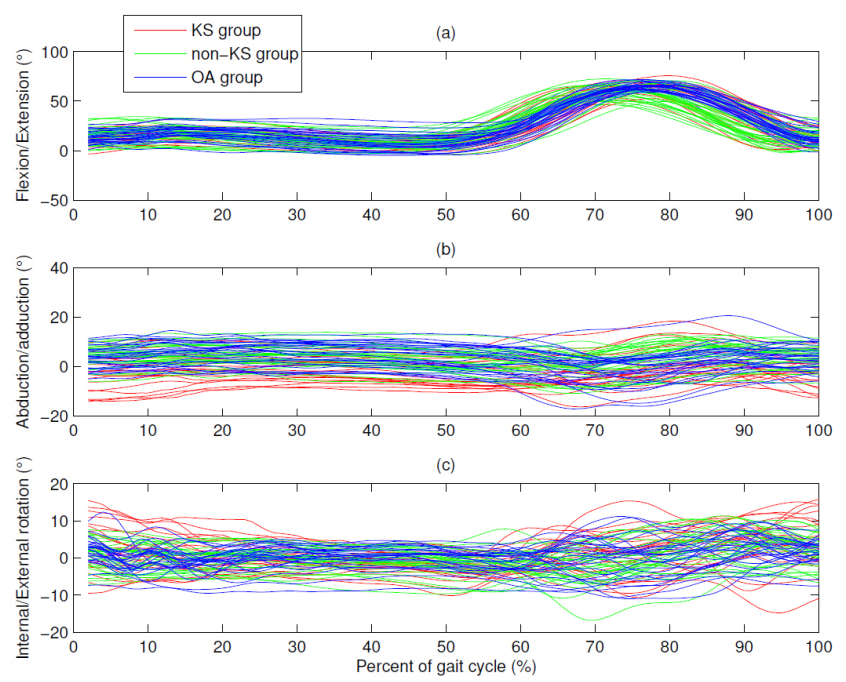

Figure 1: Kinematic gait signals (of the database) during a gait cycle: (a) Flexion/extension, (b) Abduction/adduction, and (c) Internal/external rotation. The signals were interpolated and resampled from $1 \%$ to $100 \%$ (100 points) of the gait cycle.

where,

$$
p(\mathrm{x})=\sum_{i=1}^{2} p\left(\mathrm{x} \mid \omega_{i}\right) p\left(\omega_{i}\right)
$$

$p\left(\mathrm{x} \mid \omega_{i}\right)$ is the likelihood of $\omega_{i}$ with respect to $\mathrm{x}$. It is the conditional probability of obtaining the observed measurement $\mathrm{x}$ given that it has been sampled from group $\omega_{i} \cdot p\left(\omega_{i}\right)$ is the prior probability of group $\omega_{i}$. We used the generally accepted value of OA prevalence $p\left(\omega_{2}\right)=p(O A)=15 \%$ published in the literature ${ }^{[20,21]}$ for an age group similar to that of cohort. Thus, $p\left(\omega_{1}\right)=p($ non $-K S)=85 \% \cdot p(\mathrm{x})$, which is the evidence, can be viewed as a scale factor that guarantees that the posterior probabilities sum to one. Using the Bayes formula, we have the following decision rule:

$$
\begin{aligned}
& \text { If } p\left(\omega_{1} \mid \mathrm{x}\right) \leq p\left(\omega_{2} \mid \mathrm{x}\right) \text { then decide } \mathrm{x} \in \omega_{2} \text { else, decide } \\
& \qquad \mathrm{x} \in \omega_{1},
\end{aligned}
$$

or, equivalently:

If $p\left(\mathrm{x} \mid \omega_{1}\right) p\left(\omega_{1}\right) \leq p\left(\mathrm{x} \mid \omega_{2}\right) p\left(\omega_{2}\right)$ then decide $\mathrm{x} \in \omega_{2}$ else, decide $\mathrm{x} \in \omega_{1}$.

The class-conditional densities $p\left(\mathrm{x} \mid \omega_{i}\right)$ are modelled by multivariate normal distributions:

$$
p\left(\mathrm{x} \mid \omega_{i}\right)=\frac{1}{(2 \pi)^{d / 2}\left|\Sigma_{i}\right|^{1 / 2}} e^{\left[-\frac{1}{2}\left(\mathrm{x}-\mu_{i}\right)^{t} \Sigma_{i}^{-1}\left(\mathrm{x}-\mu_{i}\right)\right]}
$$

where $i=\{1,2\}, \mu_{i}$ is the mean vector, and $\Sigma_{i}$ the covariance matrix of the class $\omega_{i}$. The density parameters are estimated from non-test data sets.

The normality of the densities is verified using the Q-Q Plot (Quantiles-Quantiles Plot) which is a plot of the percentiles of a standard normal distribution against the corresponding percentiles of the observed data. ${ }^{[22]}$ The basic assumption is that the OA and non-KS training data sets are sufficiently representative of the OA and non-KS classes.

Bayes classification seeks an optimal division of data into two classes. The normality assumption is convenient to handle mathematically, and, in our case it was verified by the Q-Q Plot. The results, to be discussed in Section 3, confirm that the normality assumption is sufficient, so that there is no need to developp and study another model.

\subsection{Statistical analysis}

An analysis of variance was performed to verify the group homogeneity. A Post hoc Tukey test was used to examine the differences between pairs of groups. The implementation of this statistical processing was done via SPSS 18.0 (Statistical Package for Social Sciences). A $P$-value of .05 was set as the criterion for statistical significance.

\section{Results}

The Q-Q Plot shown (see Figure 2), confirms that the densities corresponding to each angle data, i.e., the flexion/extension (red points), internal/external rotation (blue points), and abduction/adduction (green points), can be effectively modelled by multivariate normal distributions. 


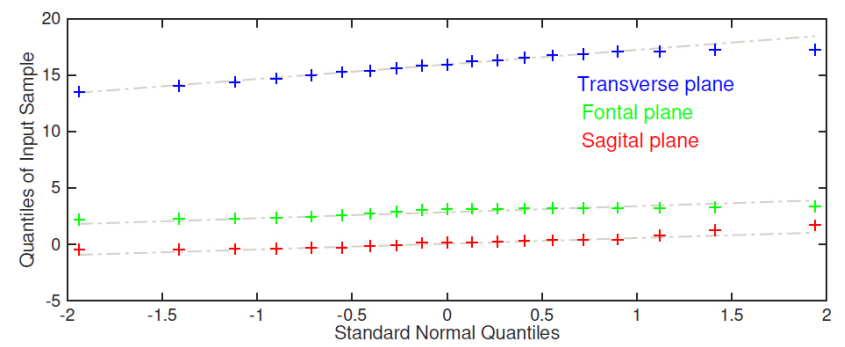

Figure 2: Normal Q-Q plot of a sample of the 25 AS subjects showing that each angle data follows a multivariate normal distribution

Table 1 shows the results of comparing KS workers data into either OA or non-KS workers. The first row displays the similarity results when the three planes data are used together by concatenation into a single vector. The other three rows are for each of the plane data separately, namely the frontal (Abduction/adduction), sagittal (Internal/external rotation), and transverse (flexion/extension) planes.

Table 1: Number of KS workers classified into non-KS worker class versus of those assigned to the knee OA class.

\begin{tabular}{lll}
\hline Plane angle data & non-KS class & knee OA class \\
\hline The three planes & 4 & 20 \\
Frontal plane & 2 & 22 \\
Sagittal plane & 15 & 9 \\
Transverse plane & 8 & 16 \\
\hline
\end{tabular}

Note. The classification is based on the Bayes decision rules using the three planes together (line 1) and each of the plane data separately (lines 2-4).

Table 2: A comparison of general subject characteristics.

\begin{tabular}{llll}
\hline & KS workers & Non-KS workers & Knee OA \\
\hline $\begin{array}{l}\text { Number of } \\
\text { subjects }\end{array}$ & 24 & 25 & 29 \\
$\begin{array}{l}\text { Age (year) } \\
\text { Percentage of } \\
\text { male/female }\end{array}$ & $47.7 \pm 6^{\dagger}$ & $52.6 \pm 9.4^{\dagger}$ & $63.3 \pm 8.4^{* \#}$ \\
$\begin{array}{l}\text { Weight }(\mathrm{kg}) \\
\text { Height }(\mathrm{cm})\end{array}$ & $75.6 \pm 13.1$ & $71.2 \pm 12.3^{\dagger}$ & $80.5 \pm 18.3^{\#}$ \\
BMI $\left(\mathrm{kg} / \mathrm{m}^{2}\right)$ & $25.8 \pm 3.3^{\dagger}$ & $23.8 \pm 2.8^{\dagger}$ & $160 \pm 10.1$ \\
\hline
\end{tabular}

Note. Significance during post hoc $t$-tests is denoted by letters representing which group was compared (*, KS workers; \#, Non-KS workers; $\dagger$, Knee OA). Significance was set at $p<0$ :05.

To insure participation at large, all the subjects who took part in this study were recruited using advertisements in local newspapers. As a result, the general subject characteristics were not matched. The Post hoc analysis of these characteristics revealed that there were some significant differences (see Table 2) between groups. The Knee OA group is older than the Non-KS workers and the Knee OA group $(p<.05)$. Also, the BMI data analysis show a significant difference between the Knee OA group compared to the Non-KS workers and the Knee OA group. However, there was no significant difference in the height variable of the three groups.

\section{Discussion}

The Bayes decision rule applied to the data from the three planes taken together (first row of Table 1) indicated that 20 KS workers $(83.3 \%)$ are more similar to the OA group and that the remaining $4 \mathrm{KS}$-workers' patterns resembled more non-KS gait patterns. This clearly indicates that the kinematics of workers exposed to knee straining postures generally resembles the kinematics of OA patients. This can be observed despite radiologically confirmed osteoarthritis in the diagnostic for these workers. This justifies further prospective studies to demonstrate if $\mathrm{KS}$-workers have a gait pattern at risk of developing the disease soon. This is consistent with studies reported in the literature. For instance, the association between work load and increased risk of knee OA was demonstrated in Ref. ${ }^{[23,24]}$ Also, a physically heavy workload was shown to be detrimental to the knee joint and to increase the risk of severe OA. ${ }^{[7]}$ Finally, it was shown that prolonged or repeated knee bending is a risk factor for knee OA, and that risk may be higher in jobs involving knee bending and mechanical loading. ${ }^{[4]}$

We also performed a focused analysis using each plane data separately to see which, if any, corroborates the conclusion of the three plane data analysis. By determining that abduction/adduction pattern of 22 out of $24 \mathrm{KS}$ workers resembles more abduction/adduction pattern of OA subjects (see Table 1), the abduction/adduction data (frontal plane) analysis alone affords the same conclusion as the three plane data analysis: the abduction/adduction kinematic data of workers exposed to knee straining postures resemble those of OA subjects (Table 1). This result is also consistent with studies reported in the literature. ${ }^{[25]}$ Particularly, it was shown in a study about the role of knee alignment (varus/valgus) in disease progression and functional decline in knee osteoarthritis, that the varus alignment at baseline increased risk of subsequent medial osteoarthritis progression and the valgus alignment at baseline increased risk of subsequent lateral osteoarthritis progression. Moreover, it was shown in a comparative study of KS workers versus non-KS workers, ${ }^{[15]}$ that KS workers have a greater foot contact angle than the non-KS workers and that they seemed to stay adducted during the entire gait cycle, particularly during the stance phase. Finally, knee adduction has been shown to correlate with high adduction moments, ${ }^{[26,27]}$ which can lead to excessive loading on the medial compartment and medial knee OA.

The analysis in the two other planes, i.e., flexion/extension and internal/external rotation, showed, on the contrary, that KS workers' knee movement in these planes did not clearly resemble the patterns of either OA or non-KS workers. These results indicate that a prospective gait analysis on risk factors related to knee straining activities should focus on a detailed analysis in all three planes of movement.

The statistical analysis confirms that the similarity found between KS workers and knee OA patients is due to their knee 
kinematic data and not to their general subject characteristics, since, the Post hoc Tukey test reveals significant statistical difference of the age and BMI between the KS worker group and the Knee OA group (see Table 2). The percentages of female to male in the OA patients and non-KS subjects are comparable ( $42 \%$ female vs. $58 \%$ male and $52 \%$ female vs. $48 \%$ male, respectively), so that there was no specific additional analysis we needed to do on the possible effect of gender.

\section{Conclusion}

In this study, we investigated a novel method based on kinematic gait signal analysis and Bayes decision rule which found that knee kinematic data resulting from knee overloading exposure strongly resemble those of knee OA patients. This emphasizes the necessity of a safe working environment for workers who are exposed to physical load. This result is consistent with and complements earlier epidemio- logical investigations using a kinematic gait signal analysis. The method offers additional insight into the mechanisms that might contribute to knee OA development and progression and thus contribute to early detection of the diseases and management of modifiable risk factors. It also justifies further prospective studies of KS workers in order to assess if gait pattern could be modified even before the onset of the disease.

\section{Acknowledgements}

The authors would like to thank the Natural Sciences and Engineering Research Council (NSERC), the CIHR Clinical Initiative Strategy program, and to the Quebec rehabilitation network (REPAR) in partnership with the Institut de recherche Robert-Sauv en sant et en scurit du travail (IRSST). Special thanks to Gerald Parent and Alexandre Fuentes for technical support and to Etienne Turcotte and Enrique Villalobos for their help in data collection.

\section{References}

[1] Issa SN, Sharma L. Epidemiology of osteoarthritis: An update. Current Rheumatology Reports. 2006; 8(1): 7-15. http://dx.doi .o $\mathrm{rg} / 10.1007 / \mathrm{s} 11926-006-0019-1$

[2] Liikavainio T. Biomechanics of Gait and Physical Function in Patients with Knee Osteoarthritis Thigh Muscle Properties and Joint Loading Assessement. Faculty of Health Sciences, University of Eastern Finland; 2010.

[3] Felson DT, Niu J, Clancy M, et al. Effect of recreational physical activities on the development of knee osteoarthritis in older adults of different weights: the Framingham Study. Arthritis Rheum. 2007; 57(2): 1-2.

[4] Cooper C, Mcalindon T, Coggon D, et al. Occupational activity and osteoarthritis of the knee. Annals of the Rheumatic Diseases. 1994; 53: 90-93. http://dx.doi.org/10.1136/ard.53.2.90

[5] Bernard TE, Wilder FV, Aluoch M, et al. Job-related osteoarthritis of the knee, foot, hand, and cervical spine. Journal Occupational Environmental Medicine. 2010; 52(1): 33-38. PMid:20042887. http://dx.doi.org/10.1097/JOM.0b013e3181c40e98

[6] Klubmann A, Gebhardt H, Liebers F, et al. Individual and occupational risk factors for knee osteoarthritis - Study protocol of a case control study. BMC Musculoskeletal Disorders. 2008; 9(1): 26. PMid:18302740. http://dx.doi.org/10.1186/1471-247 4-9-26

[7] Manninen P, Heliovaara M, Riihimaki H, et al. Physical workload and the risk of severe knee osteoarthritis. Scand J Work Environ Health. 2002; 28(1): 25-32. http://dx.doi.org/10.5271/sjw eh. 643

[8] Ainsworth BE, Leon AS, Richardson MT, et al. Accuracy of the college alumnus physical activity questionnaire. Journal of Clinical Epidemiology. 1993; 46(12): 1403-1411. http://dx.doi.org/1 $0.1016 / 0895-4356$ (93) 90140-V

[9] Washburn RA, Montoye HJ. The Assessment Of Physical Activity By Questionnaire. American Journal of Epidemiology. 1986; 123(4): 563-576. PMid:3513548.

[10] Lustig S, Magnussen R, Cheze L, et al. The KneeKG system: a review of the literature. Knee Surgery, Sports Traumatology, Arthroscopy. 2011: 1-6. PMid:21052981.
[11] Messier J, Kalaska JF. Comparison of variability of initial kinematics and endpoints of reaching movements. Experimental Brain Research. 1999; 125: 139-152. http://dx.doi.org/10.1007/s00 2210050669

[12] Al-Zahrani KS, Bakheit AMO. A study of the gait characteristics of patients with chronic osteoarthritis of the knee. Disability and Rehabilitation. 2002; 24(5): 275-280. http://dx.doi.org/10.1080 /09638280110087098

[13] Deluzio KJ, Astephen JL. Biomechanical features of gait waveform data associated with knee osteoarthritis: An application of principal component analysis. Gait and Posture. 2007; 25(1): 86-93. PMid:16567093. http://dx.doi.org/10.1016/j.gai tpost. 2006.01 .007

[14] Mezghani N, Ouakrim Y, Fuentes A, et al. knee osteoarthritis assessment using knee kinematic data classification. In: Osteoarthritis, Cartilage, editors. 2012 World Congress on Osteoarthritis (OARSI). 2012.

[15] Gaudreault N, Hagemeister N, Poitras S, et al. Comparison of knee gait kinematics of workers exposed to knee straining postures to those of non-knee straining workers. Gait and Posture. 2013; 38(2): 187-191. PMid:23206900. http://dx.doi.org/10.1016 /j.gaitpost.2012.11.004

[16] Kajaks T. The Effect of Sustained Static Kneeling on Knee Joint Gait Parameters. Queen's University, Kingston; 2008.

[17] Mezghani N, Husse S, Boivin K, et al. Hierarchical analysis and classification of asymptomatic and knee osteoarthritis gait patterns using a wavelet representation of kinetic data and the nearest neighbour classifier. Journal of Mechanics in Medicine and Biology. 2008; 8(1): 45-54. http://dx.doi.org/10.1142/S02195194 08002474

[18] Kadaba M, Ramakrishnan H, Wootten M, et al. Repeatability of kinematic, kinetic, and electromyographic data in normal adult gait. Journal of orthopedic reasearch. 1989; 6: 849-860. PMid:2795325. http://dx.doi.org/10.1002/jor.1100070611

[19] Boivin K. Dveloppement d'une approche d'valuation clinique de la cinmatique tridimensionnellle du genou durant la marche pour des patients gonarthrosiques. Institut de gni biomdical, cole Polytehnique de Montral; 2010. 
[20] Lawrence RC, Felson DT, Helmick CG, et al. Estimates of the prevalence of arthritis and other rheumatic conditions in the United States: Part II. Arthritis Rheumatism. 2008; 58(1).

[21] Helmick CG, Felson DT, Lawrence RC, et al. Estimates of the prevalence of arthritis and other rheumatic conditions in the United States: Part I. Arthritis Rheumatism. 2008; 58(1).

[22] Kim K, Timm N. Univariate and Multivariate General Linear Models: Theory and Applications Using SAS Software. Boca Raton: Chapman and Hall/CRC; 2007.

[23] Toivanen AT, Helivaara M, Impivaara O, et al. Obesity, physically demanding work and traumatic knee injury are major risk factors for knee osteoarthritis - a populationbased study with a follow-up of 22 years. 2010; 49(2): 308-314.

[24] Andriacchi TP, Mundermann A. The role of ambulatory mechanics in the initiation and progression of knee osteoarthritis. Current opin- ion in rheumatology. 2006; 18(5): 514-8. PMid:16896293. http: //dx.doi.org/10.1097/01. bor.0000240365.16842.4e

[25] Sharma L, Song J, Felson DT, et al. The role of knee alignment in disease progression and functional decline in knee osteoarthritis. Journal of the American Medical Association. 2001; 286(2): 188-95. http://dx.doi.org/10.1001/jama.286.2.188

[26] Barrios JA, Higginson SJ, Royer DT, et al. Static and dynamic correlates of the knee adduction moment in healthy knees ranging from normal to varus-aligned. Clinical Biomechanics. 2009; 24(10): 850-854. PMid:19703728. http://dx.doi.org/10.10 16/j.clinbiomech.2009.07.016

[27] Teixeira LF, Olney SJ. Relationship between alignment and kinematic and kinetic measures of the knee of osteoarthritic elderly subjects in level walking. Clinical Biomechanics. 1996; 11(3): 126-134. http://dx.doi.org/10.1016/0268-0033(95 )00057-7 